\title{
Investigation of the AODV And the SDWCA QoS Handling At Different Utilisation Levels In Adaptive Clustering Environments
}

\author{
Al-Baadani, Faris., Yousef, S., Tapaswi, S., Patnaik, K. K., and Cole, M \\ Faculty of Science \& Technology \\ Anglia Ruskin University \\ Chelmsford, Essex, UK \\ faris.al-baadani@student.anglia.ac.uk \\ sufian.yousef@anglia.ac.uk
}

\begin{abstract}
A simulation study using NS2 simulator using two main routing protocols with specific design parameters has been carried out to investigate the QoS main parameters such as throughput, delay, Jitter, Control Overhead, Number of packets, number of packets dropped and the rating overheads. The traffic is made of CBR slow video traffic. From the result it is noted that the AODV routing protocol outperforms the SDWCA routing protocols in the throughput, the delay and the jitter issues at different loading levels.
\end{abstract}

Keywords-MANETs, Clustering, Cluster-Head, WCA, AODV, Throughput, Average Delay.

\section{INTRODUCTION}

Mobile Ad hoc Networks (MANETs) are gaining popularity in recent years due to widespread use of mobile devices which are used in many real time applications. MANETs consist of wireless mobile nodes forming a network in ad hoc fashion. These ad hoc networks are mainly used to connect the mobile devices in the absence of any existing network infrastructure. Mobile nodes, mainly serving as routers forward the collected information to each other in order to maintain the connection in the network. When network gets disconnected due to node failure, one of the ways to maintain the connectivity is to re-organize the network in ad hoc fashion. Re-organization of the network includes formation of different network typologies such as cluster based topology.

Cluster based topology consists of many cluster-heads which act as the routers in the wireless backbone architecture formed in ad hoc fashion. This process results in formation of many clusters in the network which consists of wireless mobile nodes connected to their respective cluster-heads. Cluster-heads are responsible for maintaining the dynamic topology of the network and allocating the available network resources to the nodes belong to each cluster-head. This process of clustering is performed in two phases: (1) Cluster formation; this phase is responsible for selection of cluster-heads among the nodes. Once clusterhead is selected, every node belonging to that cluster routes its data to cluster-head. Since nodes are mobile, there is a possibility of nodes moving from one cluster to another. This leads to the second phase of clustering, called as (2) Cluster maintenance. The process of cluster maintenance incurs message exchanges between nodes and cluster-heads. High number of message exchanges leads to the energy depletion of nodes 
which results in decreasing network lifetime. In order to reduce the computation in the cluster maintenance phase, it is advisable to choose the optimal cluster-head. In this paper, we propose a weighted cluster-head selection algorithm based on the standing deviation. In order to select the optimal cluster-head, at this stage we consider two key parameters: connectivity and distance.

\section{RELATED WORK}

Recent cluster-head selection algorithms select the cluster-heads on the basis of factors such as node's mobility, residual energy, speed and direction, degree and geographical position. These algorithms are not computationally simple. The computationally expensive algorithms are not suitable for resource constrained environments such as MANETs.

The Highest Degree Algorithm is also known as connectivity-based algorithm [3] is based on the degree of nodes which is assumed to be the number of neighbors of a given node. At the time of cluster-head formation, every node broadcast its unique network Identifier (ID). Based on the number of IDs received by a node, it calculates its degree. High degree of a node resembles to high number of neighbors it can communicate with. Node with the highest number of received Ids is selected as the cluster-head. The drawback of this algorithm lies in the mobility of nodes. Due to their mobile nature, nodes' degree in the network change with time. So the cluster-head selection has to take place many times which consumes the network resources such as energy. Moreover, there is no upper bound on the number of nodes in a cluster.

On the other hand, another approach named as The Lowest-Identifier (LID) also known as identifier based clustering algorithm [4] chooses the node with the lowest ID as a cluster-head. Results have shown that the system performance of LID is better than Highest-Degree in terms of throughput. The drawback of this approach is its biased nature towards nodes with small degrees which may lead to faster energy depletion of nodes. Moreover this approach fails to distribute the load uniformly across the nodes.

The Distributed Clustering Algorithm (DCA) [6, 7] and Distributed Mobility Adaptive Clustering Algorithm (DMAC) are weight based approaches. According to these algorithms, a weight is assigned to each node based on its capabilities of becoming a cluster-head. Node with the highest weight is selected as clusterhead. In DCA it is assumed that network topology does not change during the execution of the algorithm. The algorithm performs better than Highest-Degree and Lowest-Identifier algorithms as far as number of messages are concerned. Due to the mobility of nodes, assigned weights change with time which results in excessive computation in cluster-head formation phase. Moreover, system parameters such as throughput and power control are not optimized.

WCA is another weight based clustering approach in which several parameters such as mobility, transmission power and degree difference are considered in order to perform clustering and selection of the cluster head. In order to equally distribute the load on all cluster-heads, a threshold on the number of nodes in a cluster is predefined. A node in the cluster can be either ordinary node or cluster-head. Cluster-head has more responsibility as compared to the ordinary nodes belonging to that cluster. Thus it is required to select the cluster-head optimally while considering its residual energy along with the other parameters such as mobility, transmission power and degree difference. Based on these parameters, each node is assigned a weight and the node with the optimum weight is elected as the cluster head. The cluster head election algorithm terminates once all the nodes become either a cluster head or a member of a cluster head. This algorithm is both mobility and scalability aware [2-2]. 


\section{PROPOSED METHODOLOGY}

NS2 which is the common simulation software has been used in this paper to perform the simulation experiments. The source of traffic which has been used for this study is the CBR (Continuous Bit Rate). The number of nodes has been used was 50 nodes which were randomly deployed in a square space of $800 * 800$ meters over the network.

In this study, it was essential that the starting and ending point of each node were located randomly. Once the final destination has been reached, the node concern node can rest and following the pause-time, the next random end-point is nominated. During the course of simulation, this is repetitive, which result in having continuous variation in the topology of the whole network. Table 1 blow shows the Simulation Parameters which were undertaken in these experiments.

\begin{tabular}{|c|c|}
\hline Parameter Name & Value \\
\hline Routing Protocols & AODV, SDWCA \\
Mobility Model & Random Waypoint \\
Simulation time & 500 second \\
Number of nodes & 50 nodes \\
Simulation area & $800 \times 800$ \\
Transmission range & $250 \mathrm{~m}$ \\
Traffic Type & CBR \\
Packet Size & 512 bytes \\
Bandwidth & 2Mbps \\
Simulator & NS2 \\
\hline \multicolumn{2}{|c|}{ Table 1-Simulation Parameters }
\end{tabular}




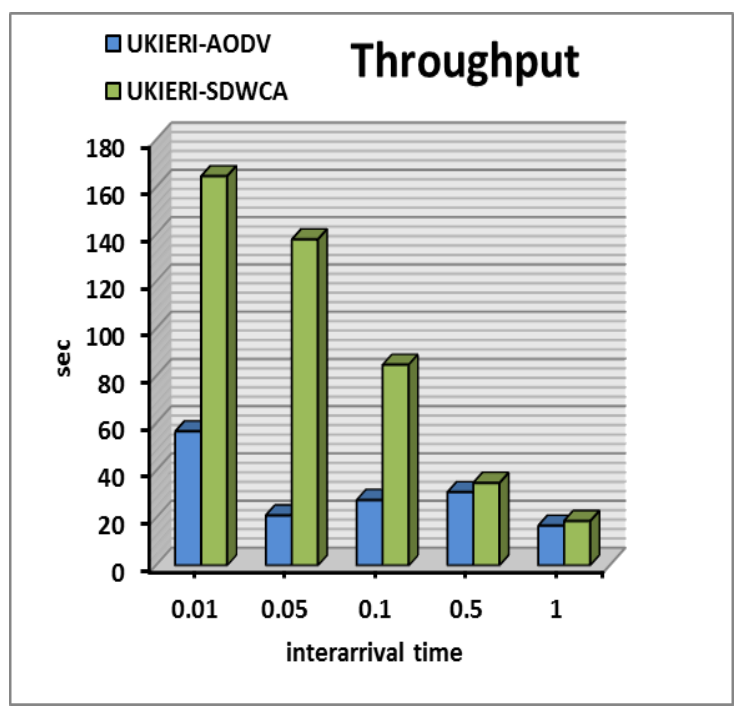

Figure 1A: Throughput

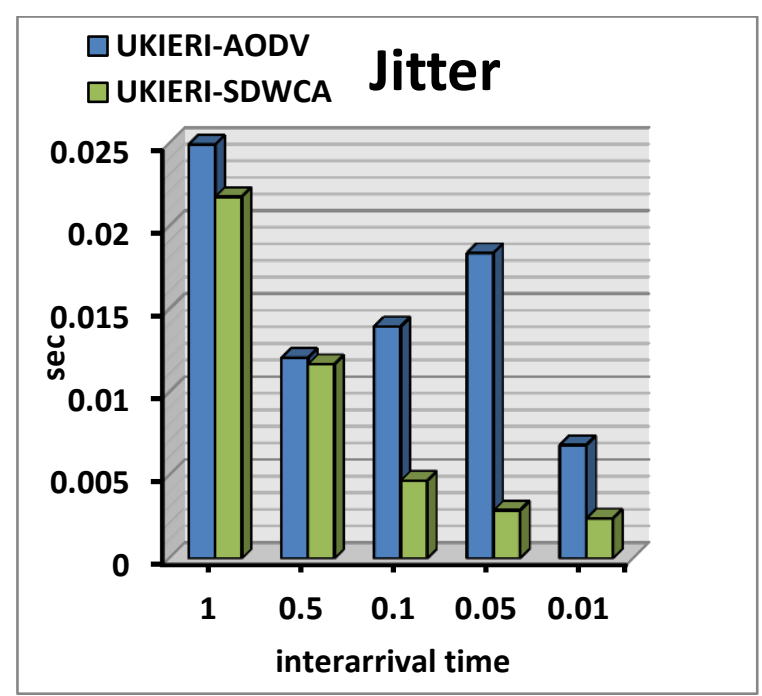

Figure 1C: Jitter

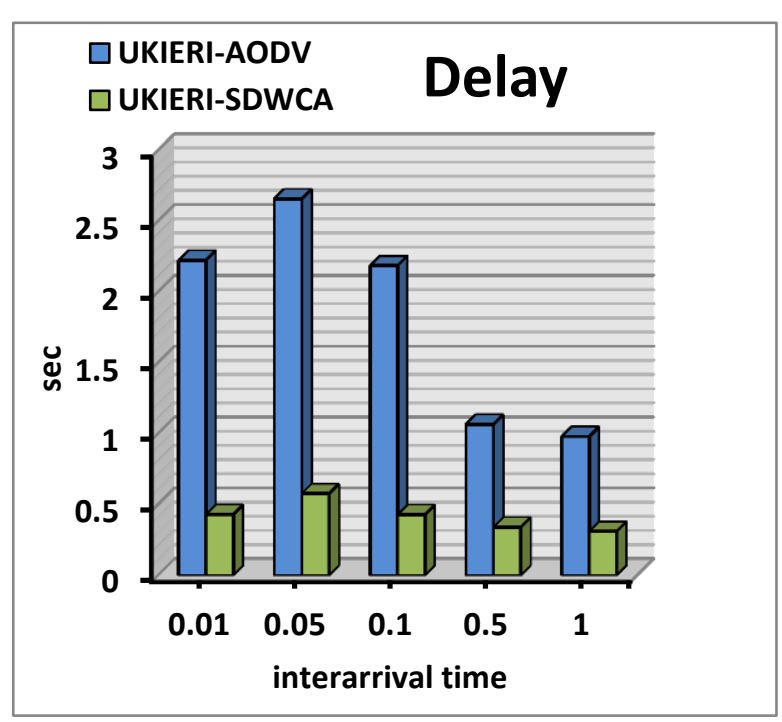

Figure 1B: Delay

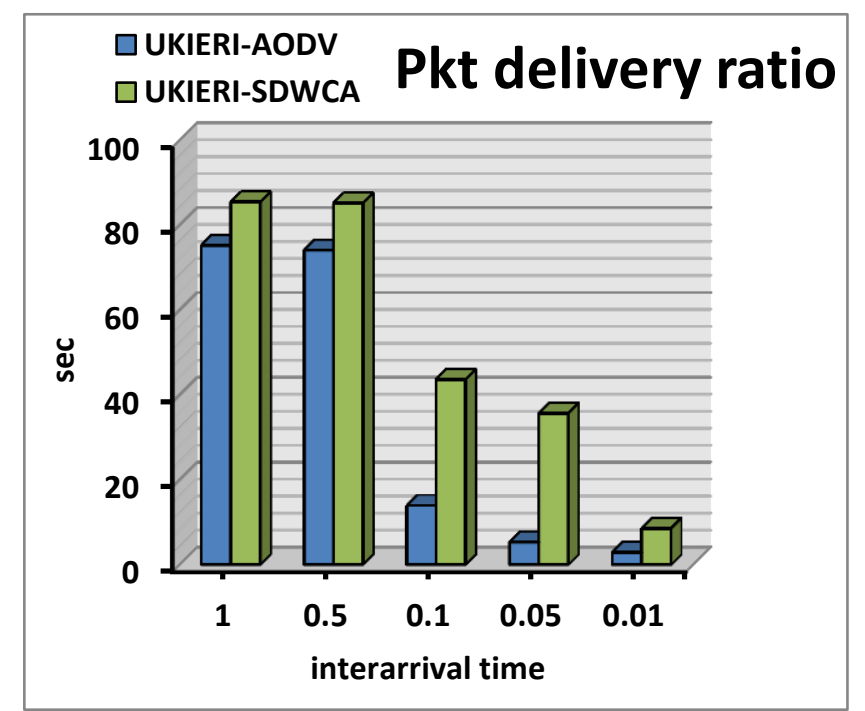

Figure 1D: Pkt Delivery Ratio 


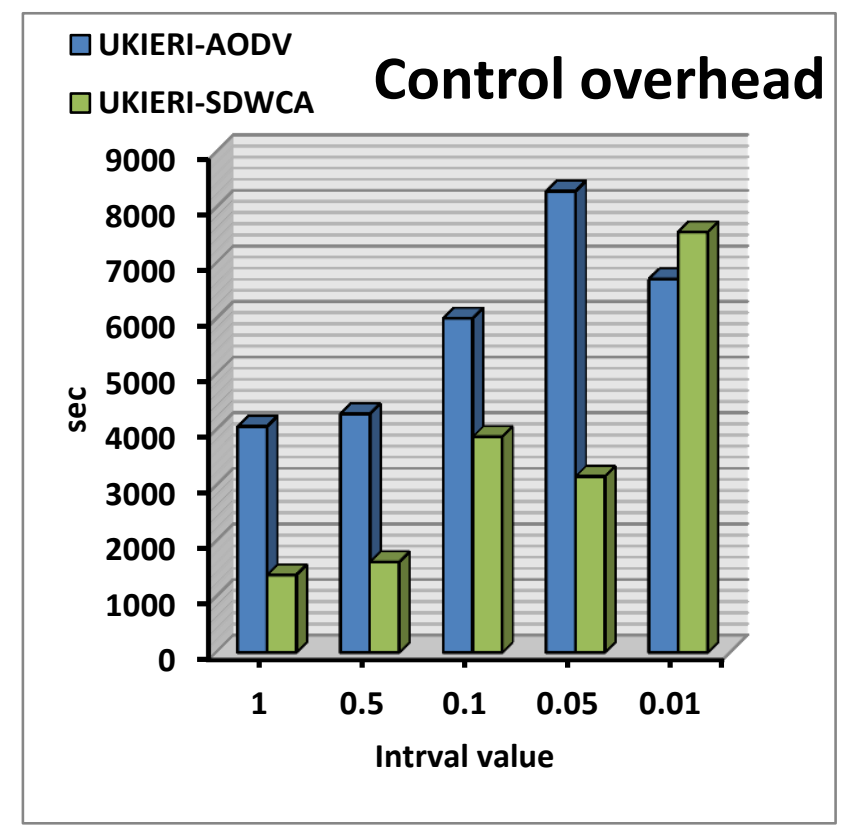

Figure 1E: Control overhead

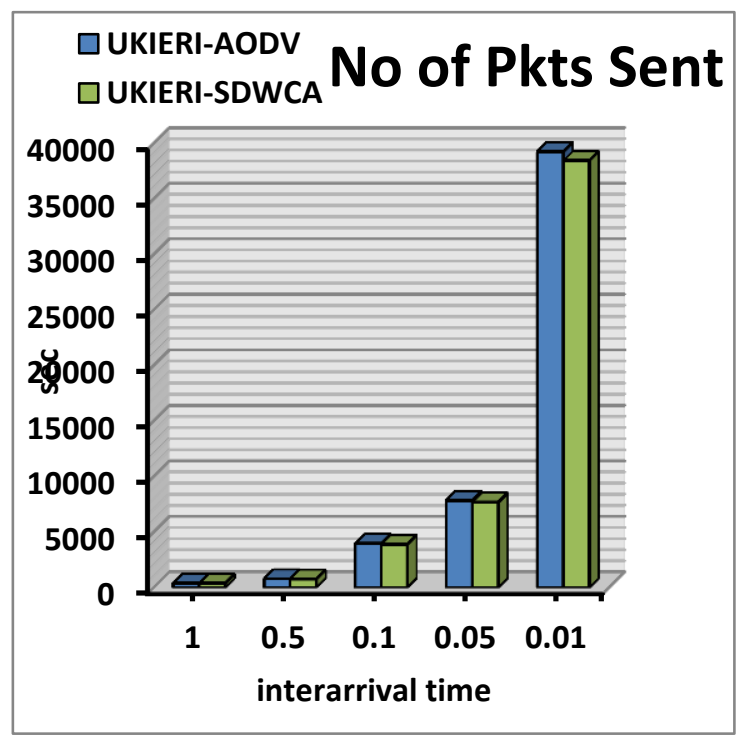

Figure 1G: No of Pkts Sent

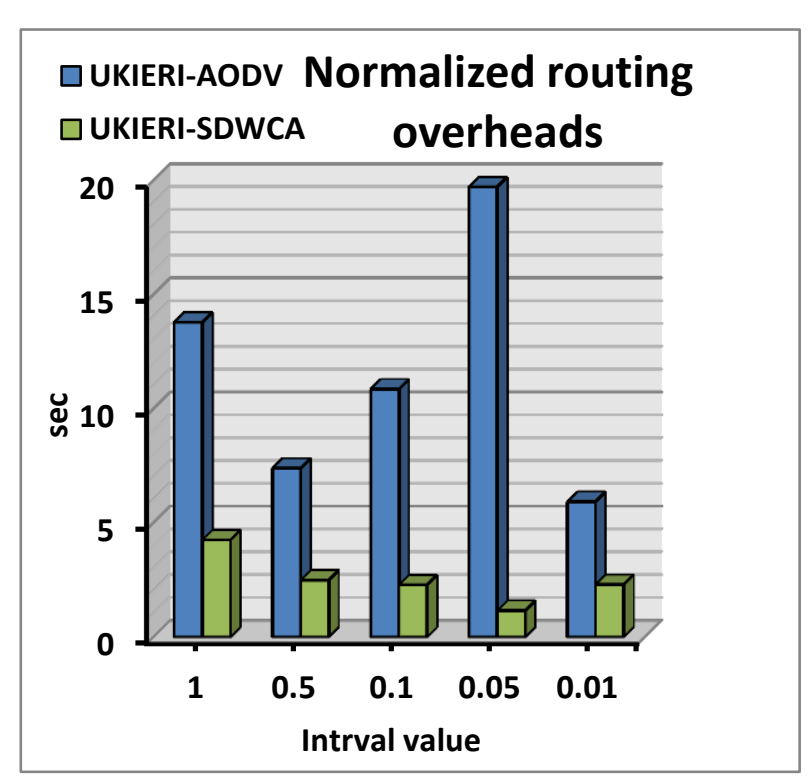

Figure 1F: Normalized routing overheads

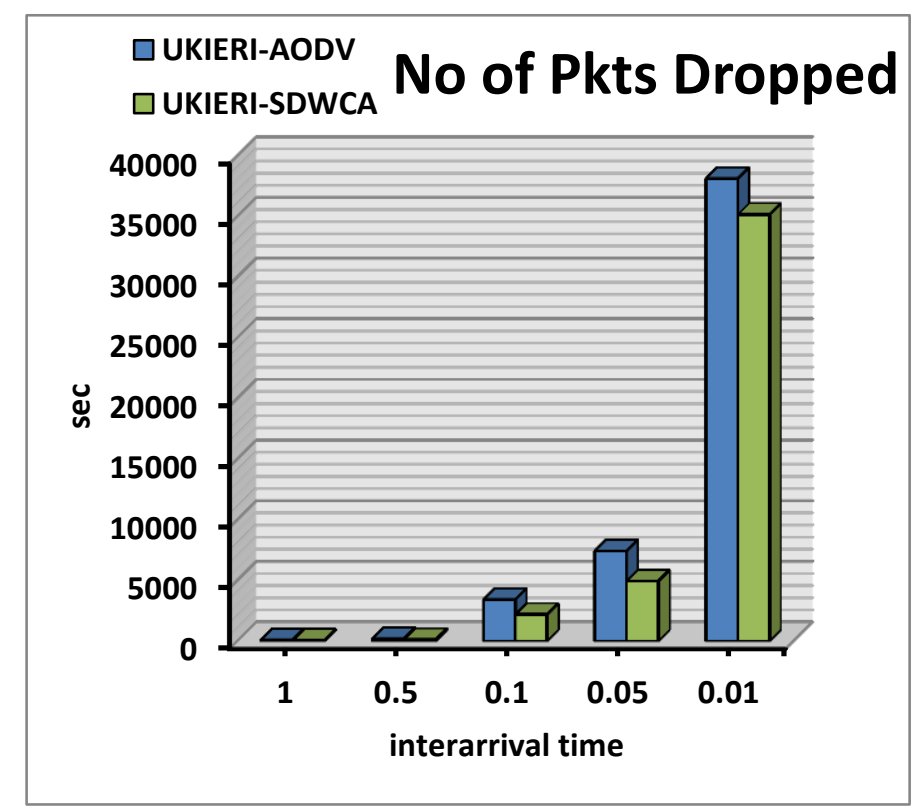

Figure 1H: No of Pkts Dropped 
Figure 1 shows various analyses using the UKIERI SDWCA to investigate effects on interval values. Two systems were used in each analysis; UKIERI-AODV (blue), UKIERI- and UKIERI-SDWCA (green).

Figure 1A shows the results for throughput; UKIERI-AODV shows the highest throughput of just under $60 \mathrm{sec}$ at an interval of 0.01 . The throughput changes as the interval value increases, decreasing to $20 \mathrm{sec}$ at 0.05 , rising again before finally falling to its lowest value at 1 . There is a clear consistent decrease in UKIERI-SDWCA throughput time as the interval value increases. This decrease is from just over $160 \mathrm{sec}$ down to below $20 \mathrm{sec}$ between intervals of 0.01 and 1 .

Figure 1B shows the data for Delay in these three analysis systems. Again, as observed with throughput analysis, the results for UKIERI-AODV, demonstrating a slight increase in delay at 0.05 , which decreases through to 1.0. The results for UKIERI- SDWCA show consistently low values throughout; hovering at the $0.5 \mathrm{sec}$ mark.

Figure 1C depicts the Jitter data analysis for the three aforementioned systems. Again, the results for UKIERI-AODV show the highest jitter value at an interval of 1.0, which dips and increases again at 0.05 , and finally being lowest at 0.01 . The highest Jitter value observed was 0.025 . The results for UKIERISDWCA show a consistent decrease in Jitter values across a decreasing interval scale, and being the lowest at 0.01 .

Figure 1D shows the results for the Pkt delivery ratio in each case. The results for UKIERI-AODV in this case show a consistent decrease in values across the 1.0 to 0.01 interval scale. Similarly, the same decrease is observed in UKIERI- SDWCA although the ratio values are higher than the previous two for all data points.

Figure $1 \mathrm{E}$ is the analysis of the Control Overhead. Here, we observe much higher values across the scale. The identical results for UKIERI-AODV show an increase in control overhead values between 1.0 and 0.05 , and end in a small decrease at 0.01. The highest value (shown at 0.05) is above $8000 \mathrm{sec}$, for UKIERIAODV.

Figure $1 \mathrm{~F}$ shows the normalised routing overheads analysis. The identical results for UKIERI-AODV show the lowest values at an interval of 0.01, increasing to their highest value of approximately 20 secs at 0.05 . Following this there is a generalised decrease only increasing again at an interval of 1.0. Data for UKIERISDWCA analysis shows generally consistent low values, hovering at around $3 \mathrm{sec}$ across the interval scale.

Figures $1 \mathrm{G}$ and $1 \mathrm{H}$ depict the data for 'Number of packets sent' and 'Number of packets dropped', respectively. For both of these analyses, both systems; UKIERI-AODV, and UKIERI- SDWCA show very similar values and a very similar pattern. All three systems show an increase in number of packets (increasing from 0 to $40,000 \mathrm{sec}$ ) between 1.0 and 0.01 intervals. 


\section{CONCLUSIONS}

It is noted that AODV has high performance in comparison to SDWCA for throughput and delay. Both protocols are not very artifact at jitter and packet delivery ratio. AODV is clearly superior to SDWCA at routing overhead. However, both show nearly similar performance when dealing with number of packets sent and number of packets dropped.

Acknowledgment

This research is funded by UKIERI grant number EB09/RE419.

\section{Reference}

Artail, H., Antoun, R., Fawaz, K.: CRUST: implementation of clustering and routing functions for mobile ad hoc networks using reactive tuple-spaces. Ad Hoc Netw. 7, 1064-1081 (2009)

Baker, D.J., Ephremides, A.: The architectural organization of a mobile radio network via a distributed algorithm. IEEE Trans. Commun. 29(11), 1694-1701 (1981)

Chatterjee, Mainak, Sajal K. Das, and Damla Turgut. "WCA: A weighted clustering algorithm for mobile ad hoc networks." Cluster computing 5.2 (2002): 193-204.

G. Ravi and G. Anil, “AdHoc networks,” Jmacademy of IT \& Management, vol. 1, pp. 18-22, 2011

I.G.Shayeb,A.R.HamzaHussein,andA.B.Nasoura, "Asurvey of clustering schemes for Mobile Ad-Hoc Network (MANET)," American Journal of Scientific and Industrial Research, vol. 20, pp. 135-151, 2011.

Lin, C.R., Gerla, M.: Adaptive clustering for mobile wireless networks. IEEE J. Sel. Areas Commun. 15(7), 12651275 (1997)

S. Basagni, "Distributed and mobility-adaptive clustering for multimedia support in multi-hop wireless networks," Vehicular Technology Conference, 1999. VTC 1999 - Fall. IEEE VTS 50th, Amsterdam, 1999, pp. 889893 vol.2.

S. Basagni, "Distributed clustering for ad hoc networks," Parallel Architectures, Algorithms, and Networks, 1999. (ISPAN '99) Proceedings. Fourth InternationalSymposium on, Perth/Fremantle, WA, 1999, pp. 310-315.

Soumyabrata Talapatra and Alake Roy "Mobility Based Cluster Head Selection Algorithm for Mobile Ad-Hoc Network," in International Journal of Computer Network and Information Security, pp.42-49, 2014 
